\title{
Ein Tag Fortbildung in Dermatologie - was bleibt hängen?
}

Djalali, S

Abstract: 78 Hausärzte aus dem Kanton Zürich nahmen an einer dermatologischen Fortbildung teil. Vorher und nachher bewerteten sie Hautläsionen hinsichtlich ihrer Malignität. Die Frage war, wie sehr sich die diagnostische Kompetenz der Kollegen durch die Fortbildung verbessern würde.

Posted at the Zurich Open Repository and Archive, University of Zurich ZORA URL: https://doi.org/10.5167/uzh-99315

Journal Article

Published Version

Originally published at:

Djalali, S (2014). Ein Tag Fortbildung in Dermatologie - was bleibt hängen? PrimaryCare, 14(18):288. 


\section{Ein Tag Fortbildung in Dermatologie - was bleibt hängen?}

\section{Hausärzte aus dem Kanton Zürich nahmen an einer dermatologischen Fortbildung teil. Vorher und nach- her bewerteten sie Hautläsionen hinsichtlich ihrer Ma- lignität. Die Frage war, wie sehr sich die diagnostische Kompetenz der Kollegen durch die Fortbildung ver- bessern würde.}

Im Rahmen des minSKIN-Projekts wurden erste Studienergebnisse veröffentlicht [1]. Das minSKIN-Projekt ist eine Kooperation des Instituts für Hausarztmedizin und der Dermatologischen Klinik des Universitätsspitals Zürich und untersucht, wie die diagnostische Kompetenz von Hausärzten hinsichtlich Hautläsionen verbessert werden kann. Dabei geht es nicht nur darum, die Rate richtig erkannter maligner Läsionen zu steigern, sondern auch die Rate der benignen Läsionen zu senken, die fälschlicherweise als maligne diagnostiziert werden und unnötige Exzisionen nach sich ziehen.

\section{Maligne oder benigne?}

Im ersten Teil des Projekts, dessen Ergebnisse publiziert wurden, ging es darum zu prüfen, welche Wirkung ein einzelner Fortbildungstag mit dermatologischen Vorlesungen und interaktiven Falldiskussionen auf die diagnostische Kompetenz hat. 78 Hausärzte aus dem Kanton Zürich beteiligten sich an der Studie und absolvierten vor und nach der Fortbildungseinheit einen Test. Sie bekamen Bilder von Hautläsionen vorgelegt und sollten mit Hilfe einer visuellen Analogskala angeben, wie wahrscheinlich sie die Läsion als maligne einstufen würden.

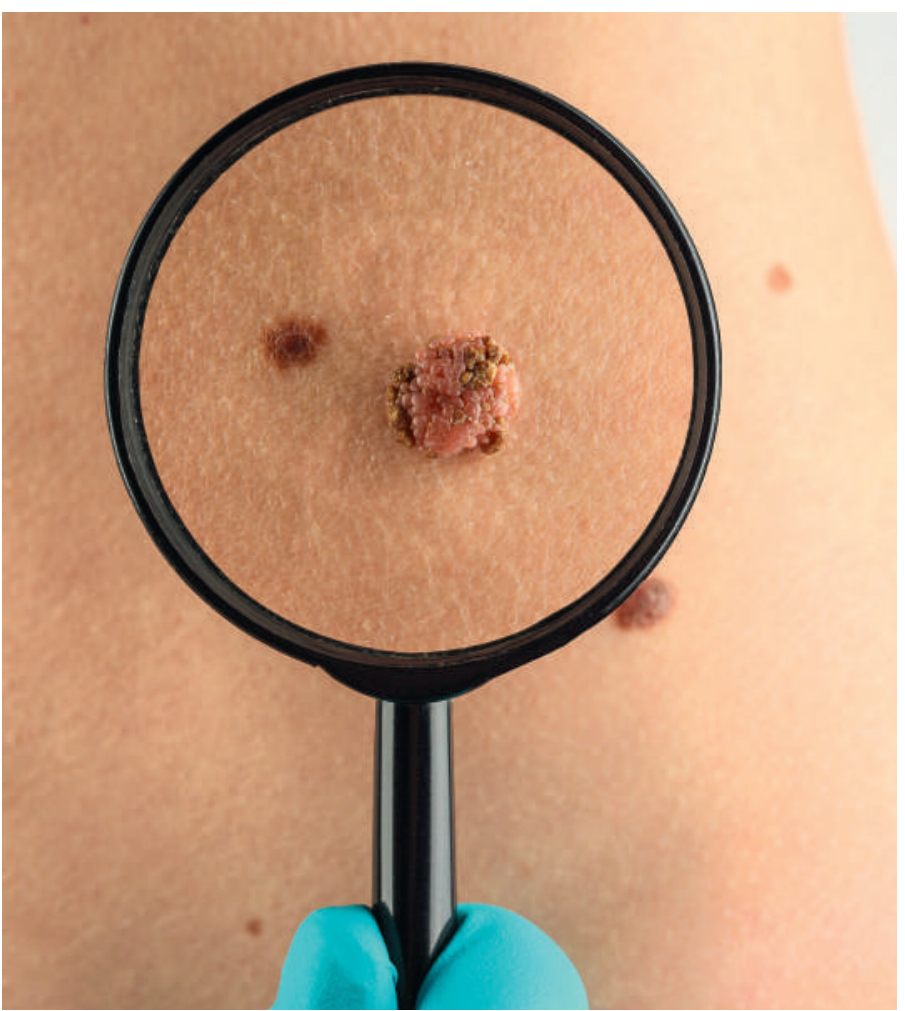

\section{Vergleich Vorher-Nachher}

Der Vorher-Nachher-Vergleich zeigt, dass die diagnostische Kompetenz der Kollegen bereits vor der Fortbildung besser war als in anderen Studien. 76,6\% der malignen Läsionen und 51,2\% der benignen Läsionen wurden korrekt erkannt. Nach der Fortbildung steigerten sich beide Raten, 82,7\% der malignen und 67,6\% der benignen Läsionen wurden korrekt erkannt - ein statistisch signifikanter Effekt.

\section{Sicherheit gewonnen}

Die visuelle Analogskala zur Bewertung der Hautläsionen war so aufgebaut, dass die Hausärzte auf einem Strahl von «sehr unwahrscheinlich maligne» bis "sehr wahrscheinlich maligne» ein Kreuz setzen mussten. Beim Test vor der Fortbildung setzten die Teilnehmer ihre Kreuze eher weiter weg von den Extrempolen als nach der Fortbildung. Daran zeigt sich, dass die Fortbildung zu mehr Sicherheitsgefühl bei der Diagnosestellung geführt hat.

\section{Langzeiteffekt fraglich}

Fortbildung und Vorher-Nachher-Tests fanden alle am selben Tag statt. Die Aussagekraft der Studienergebnisse ist daher beschränkt auf den Kurzzeiteffekt der Fortbildung. Welchen Langzeiteffekt der Tag hatte, lässt sich daraus nicht schliessen. Im weiteren Verlauf des weiterhin laufenden minSKIN-Projekts soll dieser Frage aber nachgegangen werden. Ausserdem wird der Effekt eines langfristigen Teledermatologiefeedbacks für Hausärzte untersucht. Wir dürfen gespannt sein. Bis dahin gilt: auf Fortbildung verzichten sollten wir nicht.

\section{Literatur}

1. Badertscher N, Braun RP, Held U, Kofmehl R, Senn O, et al. Diagnostic competence of Swiss general practitioners in skin cancer. Swiss Med Wkly. 2013;143: w13834

\section{Korrespondenz:}

\section{Dr. med. Sima Djalali}

Wissenschaftliche Mitarbeiterin, Institut für Hausarztmedizin Universität Zürich, Pestalozzistrasse 24, 8091 Zürich, sima.djalali[at]usz.ch, www.hausarztmedizin.uzh.ch

\section{PrimaryResearch - das Fenster zur Forschung}

In dieser Artikelserie stellen wir Forschungsarbeiten aus dem Institut für Hausarztmedizin der Universität Zürich (IHAMZ) vor. Die Originalarbeiten sind entweder «open access» zugänglich oder beim jeweiligen Autor auf Anfrage erhältlich. Die Ergebnisse geben einen spannenden Einblick in die täglichen Herausforderungen, aber auch die Leistung der Hausarztmedizin. An dieser Stelle ein herzlicher Dank an alle Kolleginnen und Kollegen, die sich an den Projekten beteiligen und die hier präsentierten Ergebnisse erst ermöglicht haben!

\section{Universität} Zürich $^{\mathrm{UzH}}$

Institut für Hausarztmedizin 